\title{
Off-diagonal photonic Lamb shift in reactively coupled waveguide-resonator system
}

\author{
M. Bernard ${ }^{a, b}$, F. Ramiro-Manzano ${ }^{b}$, N. Prtljaga ${ }^{c}$, G. Pucker ${ }^{a}$, L.Pavesi ${ }^{b}$, I.Carusotto ${ }^{d}$, \\ M.Ghulinyan ${ }^{a}$ \\ ${ }^{a}$ Centre for Materials and Microsystems, Fondazione Bruno Kessler, I-38123 Povo, Italy; \\ ${ }^{b}$ Department of Physics, Nanoscience Laboratory, University of Trento, I-38123 Povo, Italy; \\ ${ }^{c}$ Department of Physics and Astronomy, University of Sheffield, S3 7RH, United Kingdom; \\ ${ }^{d}$ INO-CNR BEC Center and Department of Physics, University of Trento, I-38123 Povo, Italy
}

\begin{abstract}
We report on a joint theoretical and experimental study of an analogue of the Lamb shift in the photonic framework. The platform is an integrated photonic device consisting of a single mode waveguide vertically coupled to a disk-shaped microresonator. The presence of a neighboring waveguide induces a reactive inter-mode coupling in the resonator, an effect analogous to an off-diagonal Lamb shift from atomic physics. Waveguide mediated coupling of different radial families results in peculiar Fano lineshapes in the waveguide transmission spectra, which manifests for different relative frequency shifts of the modes at different azimuthal numbers. Finally, a non-linear model for the dinamic tuning of the Fano lineshape under continuous wave pumping conditions is proposed.
\end{abstract}

Keywords: Integrated photonics, Lamb shift, Fano resonance, Non-linear Optics

\section{INTRODUCTION}

In the theory of open systems, the study of the coupling between a physical system to an environment constitutes a central problem. ${ }^{1}$ On one hand, the dissipative component of the coupling allows to pump the system from outside and to observe the energy which is radiated through active decay channels. On the other hand, the reactive component of the coupling shifts the energy levels of the system. Most celebrated examples of this physics involve an atom coupled to the bath of electromagnetic modes, ${ }^{2}$ namely, the (dissipative) spontaneous emission of photons from an excited state ${ }^{3-5}$ and the (reactive) Lamb shift of energetic levels of transitions (Fig. 1a). ${ }^{6-8}$

First experiments in late 1970 's ${ }^{9}$ revealed that destructive interference effects between different decay paths, which leads to the same final state in the continuum, can efficiently suppress absorption by a multilevel atom. These phenomena are known as the Coherent Population Trapping (CPT) ${ }^{10}$ and Electromagnetically Induced Transparency (EIT). ${ }^{11,12}$ While originally these phenomena were discovered in the atomic physics context, analogous effects in solid-state systems,${ }^{13}$ quantum billiards, ${ }^{14-16}$ photonic devices, ${ }^{17-23}$ and, very recently, optomechanical systems ${ }^{24}$ have been in the center of a continuous interest. While typically in most experiments only the dissipative features are affected by the interference, the theory predicts that a similar phenomenon should also occur on the reactive coupling side. ${ }^{1}$

In photonics, the presence of a waveguide in the vicinity of a resonator activates radiative decay channels for the cavity modes via leakage of the electromagnetic wave into the waveguide mode. ${ }^{25-27}$ The corresponding reactive effect corresponds to a shift of the resonator mode frequencies, which can be formally interpreted as the photonic analogue of the atomic Lamb shift (Fig. 1b). Here, we report on a joint theoretical and experimental study of such a photonic system in which pairs of optical modes at very similar frequencies are coupled simultaneously to the same waveguide mode. In this case, both the dissipative and the reactive couplings of the

Further author information: (Send correspondence to M.B.)

M.B.: E-mail: bernard@fbk.eu, Telephone: +39 0461314021

Integrated Photonics: Materials, Devices, and Applications III, edited by Jean-Marc Fédéli,

Proc. of SPIE Vol. 9520, 952006 · @ 2015 SPIE · CCC code: 0277-786X/15/\$18

doi: $10.1117 / 12.2178984$

Proc. of SPIE Vol. $9520952006-1$ 

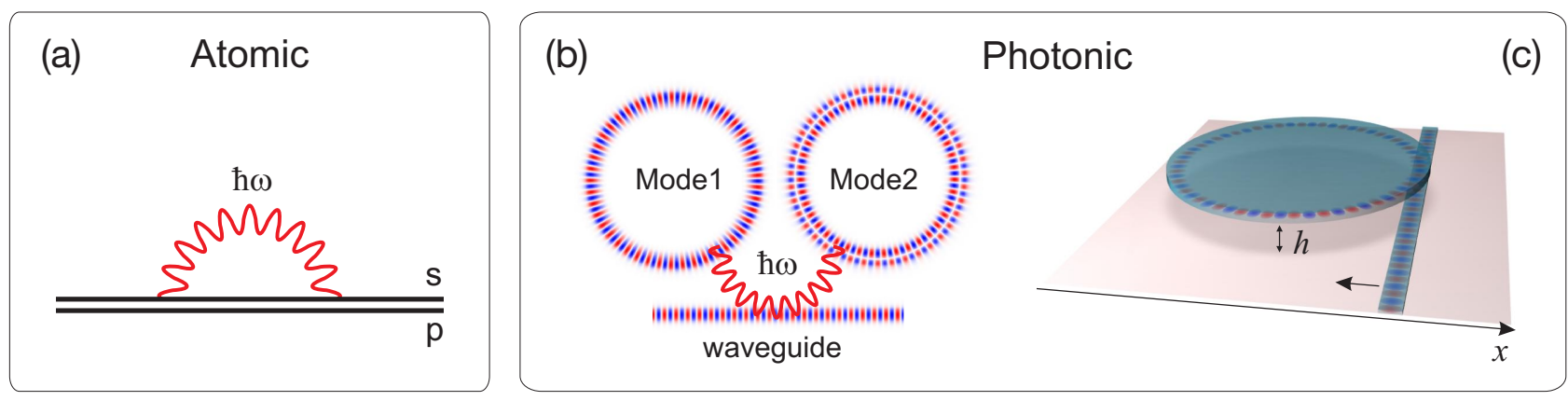

Figure 1. (a) In atomic physics, following Lamb's theory, the interaction of an atom with a bath of electromagnetic modes (the vacuum) shifts the energy levels. (b) In the photonic analogue, two eigenmodes of the same resonator may interact through a waveguide. (c) The proposed experiment is composed by a microresonator vertically coupled to an integrated single-mode waveguide located below the disk. A crucial feature is the possibility to engineer the relative coupling of different radial mode families shifting the waveguide under the resonator.

cavity modes to the waveguide turn out to be affected by interference phenomena between the resonator modes, which can be summarized as environment-induced inter-mode couplings. In analogy to the atomic phenomena, the dissipative component of the coupling gives a CPT effect, while the reactive one induces an off-diagonal Lamb shift. We show that a consideration of both of these coupling terms is necessary in order to explain the asymmetric Fano interference lineshapes observed in the transmission spectra of the system.

Moreover, we address the possibility to tune dynamically the degree of asymmetry (lineshape) of the Fano resonances under the conditions of a continuous wave pumping of the photonic device. For this, we have developed the theoretical basis which treats with cross-coupled differential equations, including non-linear optical pumping terms, for field amplitudes of the interacting resonator modes. The numerical simulations based on the dynamic model predict the necessary experimental conditions under which the Fano lineshape can be dynamically driven from large mode detunings to resonance crossover (zero detuning) condition where the EIT feature manifests.

\section{MEASUREMENTS}

\subsection{The device}

The investigated system consists of a thin microdisk resonator vertically coupled to an integrated single-mode waveguide located below the disk (Fig. 1c). While the vertical coupling is much flexible from the point of view of fabrication, it allows for an independent lateral and vertical positioning of the waveguide. In contrast to the traditional lateral coupling solution, where only the most external RMF is typically exited, the vertical coupling permits to freely tune the coupling to the different radial mode families (RMF), in particular to the more internal ones. $^{28,29}$ This comes out to be a crucial feature: since the inner RMF exhibit lower intrinsic quality factors the vertical coupling allows for several RMFs to be simultaneously close to the critical coupling and, therefore, easily observable in the waveguide transmission spectra.

\subsection{Experimental data}

The samples were tested using the experimental setup reported in Fig. 2a: a tunable C-band laser injects the light into the sample with a controlled polarization through an optical lensed fiber. The transmitted light is collected at the output facet of the waveguide using another fiber and sent to a photodetector. Figure $2 \mathrm{~b}$ reports an example of spectrum of a $40 \mu \mathrm{m}$ diameter silicon nitride microresonator. For the designed relative lateral position of the waveguide, ${ }^{28}$ the first, the second and the third radial families of the microdisk resonator are excited with different coupling constants. In the following, we will take into consideration the first and second radial families only, neglecting any possible contribution from the weak third one, provided that the spectral position of this last is far enough from the wavelength range of interest. 
(a)

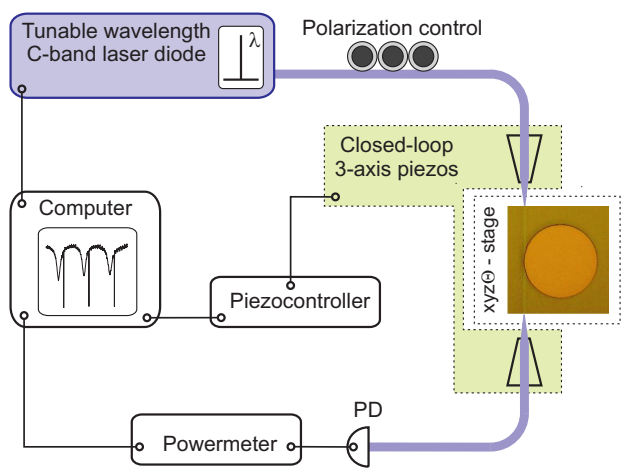

(b)

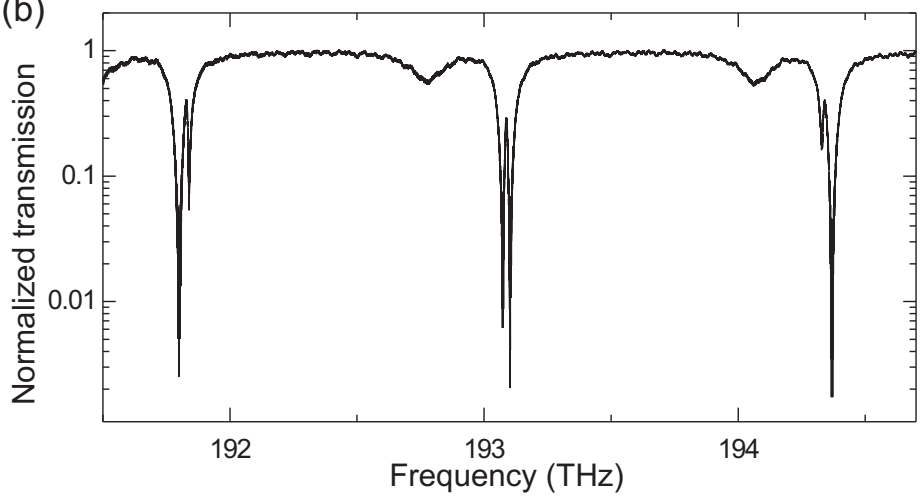

Figure 2. A sketch of the experimental setup used to measure the transmission spectra of the system is shown (a). A tunable C-band laser, filtered by a polarization controller, is injected into the waveguide with the aid of tapered fibers. Transmitted light is collected with another fiber taper and the intensity is measured by a photodiode. An example of the resulting spectra where resonances cross each other is shown in (b).

In general, different radial mode families have a different free spectral range (FSR). In this particular case, the FSR difference between the first and the second RMFs is about $20 \mathrm{GHz}$, so that when observing different azimuthal modes of one of the radial families, the peaks of the other radial family "slide" across the first one. When two different radial family modes overlap spectrally, under favorable coupling conditions, an efficient exchange of electromagnetic energy may take place between otherwise orthogonal modes. This may lead to interference phenomena where the new "hybrid" resonance takes the form of an asymmetric Fano lineshape, typical for two degenerate and interfering modes.

\section{MODEL}

The presence of a waveguide in the vicinity of a resonator builds up a channel for injecting optical power inside the resonator. However, this means also that new decay channels, namely those which emit light into the waveguide, are now present. Furthermore, following an intuitive reasoning, there should be also a reactive contribution due to the fact that in the vicinity of the waveguide the mode's surroundings have a different refractive index, which may result in a shift of the resonator mode frequencies.

In another perspective, the waveguide may be seen as a medium through which the modes interact with themselves: each mode may decay into and reabsorb a photon from the waveguide. This effect is analogous to the Lamb effect in atomic physics (Fig. 1a). In the present work, we model the interactions between the waveguide and the resonator modes with both dissipative and reactive terms. Furthermore, we introduce interference terms which may be seen as environment-induced inter-mode couplings. These terms are analogous to the coherent population trapping and to a sort of off-diagonal Lamb shift for the dissipative and reactive components, respectively.

In order to verify and quantify the mode frequency shifts, a model has to be built for comparing with the experimental data. Therefore, we write down the transmission of the whole system as a function of the mentioned shift parameters. As stated before, we will focus on a system with two modes $j=1,2$ interacting with a single (transverse) mode waveguide with a continuum of $(\mathrm{K})$ modes, although generalization to $n$ modes for the resonant system is possible. ${ }^{30}$ The transmission of a waveguide which is coupled to a resonator reads then

$$
T\left(\omega_{\mathrm{inc}}\right)=\left|E_{\mathrm{tr}} / E_{\mathrm{inc}}\right|^{2}=\left|1-i \rho \sum_{j=1,2} \bar{g}_{j}^{*} \bar{\alpha}_{j} / E_{\mathrm{inc}}\right|^{2},
$$

where $\rho=|d K / d \Omega|$ is the density of states in the waveguide, $\bar{g}_{j}^{*}$ is the coupling amplitude of the $j$ th resonator mode and $\bar{\alpha}_{j}$ are the stationary solutions for the modes which remain to be found.

In order to build up the equations of motion for the field amplitudes inside the two modes $\alpha_{j=1,2}$ let's define the symbols for the quantities we are going to use: 
$\omega_{j}^{o}$ is the center frequency of the unperturbed mode, $\Delta_{j j}$ is the diagonal Lamb-like shift which moves the mode $j$ thanks to the interaction with the waveguide, $\gamma_{j}^{\mathrm{nr}}$ represents the intrinsic losses of the resonator, while $\Gamma_{j j}^{\mathrm{rad}}$ represents the radiative losses contribution due to the coupling to the waveguide. Analogously, we define some new terms $\Delta_{12}$ and $\Gamma_{12}^{\text {rad }}$ which represent the non-diagonal Lamb-like shift and losses, respectively.

We may now write down the equation of motion for the field amplitudes inside the considered modes in the standard way, considering the new terms which may be summarized into two $\Delta$ and $\Gamma^{\mathrm{rad}}$ matrices, which can be proved to be Hermitian:

$$
i \frac{d \alpha_{j}}{d t}=\left[\omega_{j}^{o}+\Delta_{j j}-i \frac{\gamma_{j}^{\mathrm{nr}}+\Gamma_{j j}^{\mathrm{rad}}}{2}\right] \alpha_{j}+\left(\Delta_{12}-i \frac{\Gamma_{12}^{\mathrm{rad}}}{2}\right) \alpha_{3-j}+\bar{g}_{j} E_{\mathrm{inc}}(t) .
$$

\subsection{Data fitting}

Least square fitting was performed on the experimental data using the Eq.(2) in order to estimate the values of $\Gamma$ and $\Delta$. In Fig.3a an example of the experimental Fano spectrum (scatter) with the model fit (dashed line) is shown. From the fit data the $\Gamma^{\mathrm{rad}}$ and $\Delta$ matrices were extrapolated, from which the distribution of the fields may be calculated. The field intensity corresponding to the fitted data is shown in Fig.3b.

In these experiments, the Fano lineshapes for various detunings are the result of difference in the FSR of first and second RMFs, therefore, a limited number of Fano spectra with discrete detuning values are available. It would be interesting to have the possibility to continuously and arbitrarily tune the relative detuning of modes. For this, however, a different approach is needed.

\subsection{Nonlinear model}

In order to sweep the first resonance through the second, one needs to continuously tune the difference between the effective refractive index that two modes feel. This means that we need to employ an effect which changes the central frequency of the two modes by different amount. Considering the fact that often the first radial family has a much higher quality factor than the second one, non-linear effects, which rely on the intensity of the intracavity fields may be employed in order to tune the relative position of peaks and eventually scan the transition of one of the modes through the other.

(a)

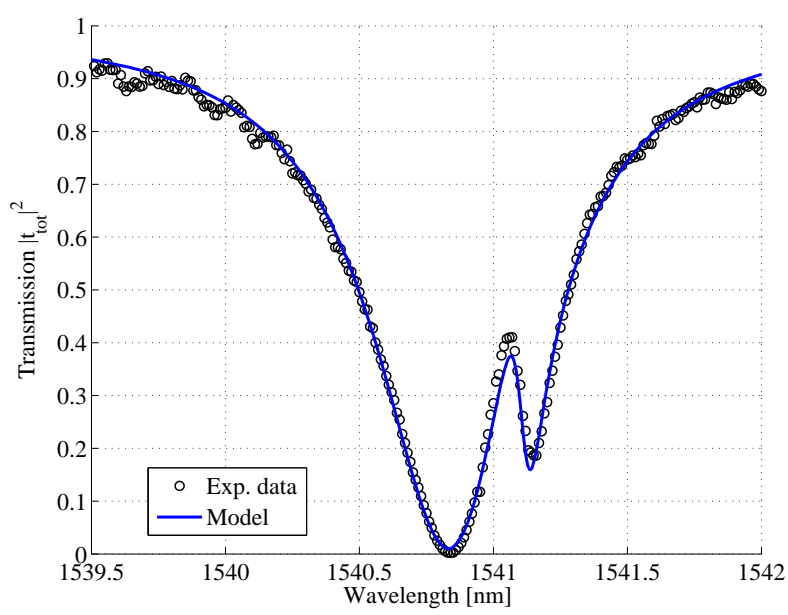

(b)

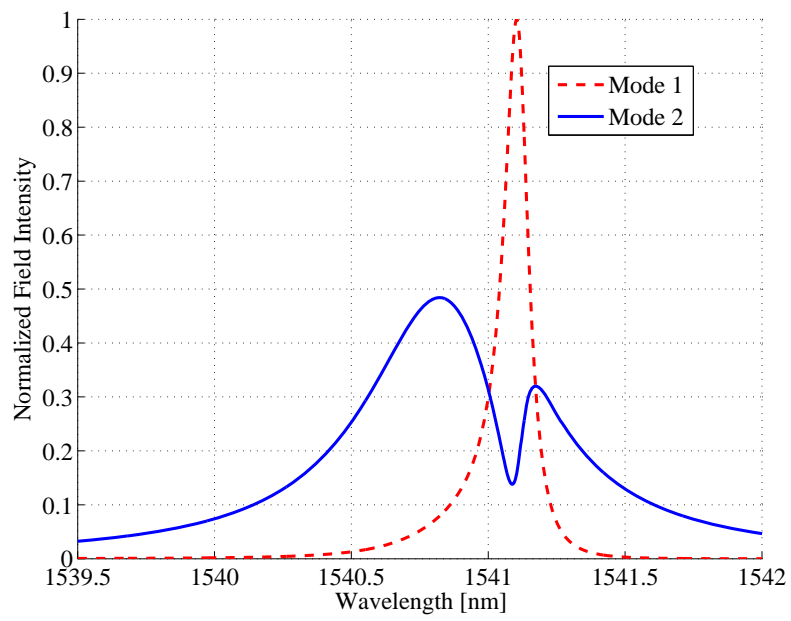

Figure 3. In panel a) an example of experimental transmission spectrum corresponding to a couple of peaks is shown (black circles), along with the resulting fit with the model of Eq. (2) (continuous blue line). Using the same model, the spectral intensity distribution was calculated (b) separately for the sharp first radial family mode (dashed red line) and the broader second radial family mode (continuous blue line). 


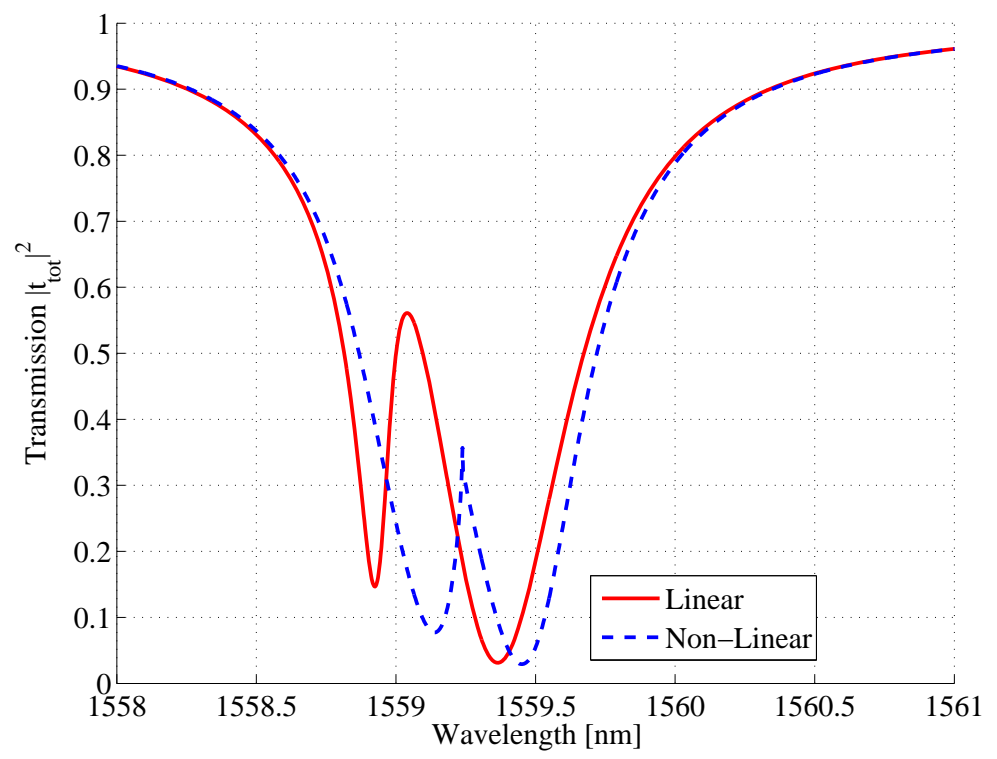

Figure 4. The transmission spectra around a couple of resonances is shown (continuous red line), following Eq.(2). With the introduction of a non-linearity continuos control of peaks detuning becomes possible according to the non-linear Eq.(4). An example of a "snapshot" solution of this equation is reported as the dashed blue line.

For this purpose, let us introduce the matrix $G$ of the non-linear coupling, whose elements have the form:

$$
g_{i, j}=g \frac{\int \epsilon(r)^{2}\left|\mathcal{E}_{i}(r)\right|^{2}\left|\mathcal{E}_{j}(r)\right|^{2} d^{3} r}{\int \epsilon(r)\left|\mathcal{E}_{i}(r)\right|^{2} d^{3} r \int \epsilon(r)\left|\mathcal{E}_{j}(r)\right|^{2} d^{3} r},
$$

where $g$ is a coupling constant, $\epsilon(r)$ is the dielectric constant of the medium and $\mathcal{E}$ is the electric field. The $G$ matrix should represent an interaction between the intensities of the modes, as it may occur during a thermal heating of cavity modes due to material absorption. The equation of motion Eq.(2), which includes the new non-linear terms, now reads as

$$
i \frac{d \alpha_{j}}{d t}=\left[\omega_{j}^{o}+\Delta_{j j}-i \frac{\gamma_{j}^{\mathrm{nr}}+\Gamma_{j j}^{\mathrm{rad}}}{2}\right] \alpha_{j}+\left(\Delta_{12}-i \frac{\Gamma_{12}^{\mathrm{rad}}}{2}\right) \alpha_{3-j}+\bar{g}_{j} E_{\mathrm{inc}}(t)+\left(g_{j j}\left|\alpha_{j}\right|^{2}+g_{12}\left|\alpha_{3-j}\right|^{2}\right) \alpha_{j},
$$

where $g_{j j}$ is the self-interaction term of the mode, while $g_{12}$ is the cross-interaction term between two modes. In general, $g_{j j}$ is expected to be larger for the first RMF which has typically a larger mode confinement factor compared to the second RMF. On the other hand, $g_{12}$ is expected to be significantly low due to the spatial (radial)l mismatch of the field intensity maxima of the two RMFs. Since the Eq. 4 is a non-linear differential equation, a numerical solver has to be employed in order to find the solutions. In Fig. 4 an example of the linear and non-linear solutions are shown as a function of wavelength. Since the nonlinearity may introduce a hysteresis, both spectra were taken considering the pump laser sweeping from short to long wavelengths. The continuous red line represents the cold-cavity situation, in which the resonator has a weak probe and the non-linear terms are therefore negligible. Increasing the power, one obtains the hot-cavity spectrum (dashed blue line). In this case both transmission deeps are shifted towards the red side of the spectrum, with the sharp first RMF showing a larger spetral displacement towards the second RMF due to the higher nonlinear coefficient.

\section{CONCLUSIONS}

In this joint theoretical and experimental study we have reported on the formation of asymmetric Fano lineshapes in the spectrum of a microdisk resonator vertically coupled to a single-mode waveguide. We revealed 
the importance of the inter-mode dissipative and reactive couplings due to the neighboring waveguide and have characterized the peculiar Fano line shapes manifesting in transmission spectra. In addition, we have developed further the static model for Fano resonances to make it feasible also for the study of dynamic effects. This model predicts that introduction of the resonator material non-linearity allows for continuous control of the relative detuning of the resonances constituting the Fano lineshape.

\section{ACKNOWLEDGMENTS}

We acknowledge financial support from the Autonomous Province of Trento, partly under the Call "Grandi Progetti 2012", project "On silicon chip quantum optics for quantum computing and secure communications SiQuro". IC acknowledges partial support from ERC via the QGBE grant. M. B., M. G. and G. P. acknowledge the support of the CMM microfabrication facility of FBK during sample fabrication.

\section{REFERENCES}

[1] Breuer, H.-P. and Petruccione, F., [The Theory of Open Quantum Systems], Oxford University Press (2002).

[2] C.Cohen-Tannoudji, J.Dupont-Roc, and G.Grynberg, [Processus d'interaction entre photons et atomes], InterEditions and Editions du CNRS (1988).

[3] Hulet, R., Hilfer, E. S., and Kleppner, D., "Inhibited spontaneous emission by a rydberg atom," Phys. Rev. Lett. 55, 2137 (1985).

[4] Yablonovitch, E., "Inhibited spontaneous emission in solid-state physics and electronics," Phys. Rev. Lett. 58, 2059 (1987).

[5] Lodahl, P., van Driel, A. F., Nikolaev, I. S., Irman, A., Overgaag, K., Vanmaekelbergh, D., and Vos, W. L., "Controlling the dynamics of spontaneous emission from quantum dots by photonic crystals," Nature 430, 654-657 (2004).

[6] Lamb, W. E. and Retherford, R. C., "Fine structure of the hydrogen atom by a microwave method," Phys. Rev. 72, 241-243 (1947).

[7] Hänsch, T. W., Shahin, I. S., and Schawlow, A. L., "Optical resolution of the lamb shift in atomic hydrogen by laser saturation spectroscopy," Nature 235, 63-65 (1972).

[8] Lempicki, A., Andrews, L., Nettel, S. J., McCollum, B. C., and Solomon, E. I., "Spectroscopy of Cr3+ in glasses: Fano antiresonances and vibronic lamb shift," Phys. Rev. Lett. 44, 1234-1237 (1980).

[9] Alzetta, G., Gozzini, A., Moi, L., and Orriols, G., "An experimental method for the observation of r.f. transitions and laser beat resonances in oriented na vapour.," Nuovo Cimento B 36, 5-20 (1976).

[10] Arimondo, E. Prog. Optics 35, 257 (1996).

[11] Boller, K.-J., Imamoglu, A., and Harris, S. E., "Observation of electromagnetically induced transparency," Phys. Rev. Lett. 66, 2593-2596 (1991).

[12] Fleischhauer, M., Imamoglu, A., and Marangos, J. P., "Electromagnetically induced transparency: Optics in coherent media," Rev. Mod. Phys. 77, 633-673 (2005).

[13] Brunner, D., Gerardot, B. D., Dalgarno, P. A., Wüst, G., Karrai, K., Stoltz, N. G., Petroff, P. M., and Warburton, R. J., "A coherent single-hole spin in a semiconductor," Science 325, 70-72 (2009).

[14] Rotter, S., Libisch, F., Burgdörfer, J., Kuhl, U., and Stöckmann, H.-J., "Tunable fano resonances in transport through microwave billiards," Phys. Rev. E 69, 046208 (2004).

[15] Mendoza, M. and Schulz, P. A., "Imaging and switching of fano resonances in open quantum cavities," Phys. Rev. B 71, 245303 (2005).

[16] Miroshnichenko, A. E., Flach, S., and Kivshar, Y. S., "Fano resonances in nanoscale structures," Rev. Mod. Mhys. 82, 2257-2298 (2010).

[17] Maleki, L., Matsko, A. B., Savchenkov, A. A., and Ilchenko, V. S., "Tunable delay line with interacting whispering-gallery-mode resonators," Opt. Lett. 29, 626-628 (2004).

[18] Xu, Q., Sandhu, S., Povinelli, M. L., Shakya, J., Fan, S., and Lipson, M., "Experimental realization of an on-chip all-optical analogue to electromagnetically induced transparency," Phys. Rev. Lett. 96, 123901 (2006). 
[19] Xiao, Y., He, L., Zhu, J., and Yang, L., "Electromagnetically induced transparency-like effect in a single polydimethylsiloxane coated silica microtoroid," Appl. Phys. Lett. 94, 231115 (2009).

[20] Dong, C., Zou, C., Xiao, Y., Cui, J., Han, Z., and Guo, G., "Modified transmission spectrum induced by two-mode interference in a single silica microsphere," J. Phys. B: At. Mol. Opt. Phys. 42, 215401 (2009).

[21] Li, B., Xiao, Y., Zou, C., Liu, Y., Jiang, X., Chen, Y., Li, Y., and Gong, Q., "Experimental observation of fano resonance in a single whispering-gallery microresonator," Appl. Phys. Lett. 98, 021116 (2011).

[22] Gu, J., Singh, R., Liu, X., Zhang, X., Ma, Y., Zhang, S., Maier, S. A., Tian, Z., Azad, A. K., Chen, H.-T., Taylor, A. J., Han, J., and Zhang, W., "Active control of electromagnetically induced transparency analogue in terahertz metamaterials," Nat. Commun. 3, 1151 (2012).

[23] Huang, Q., Shu, Z., Song, G., Chen, J., Xia, J., and Yu, J., "Electromagnetically induced transparency-like effect in a two-bus waveguides coupled microdisk resonator," Opt. Express 22, 3219-3227 (2014).

[24] Weis, S., Rivière, R., Deléglise, S., Gavartin, E., Arcizet, O., Schliesser, A., and Kippenberg, T. J., "Optomechanically induced transparency," Science 330, 1520-1523 (2010).

[25] Matsko, A. B., [Practical Applications of Microresonators in Optics and Photonics], CRC Press (2009).

[26] Lee, D. L., [Electromagnetic Principles of Integrated Optics], Wiley (1986).

[27] Yariv, A., "Universal relations for coupling of optical power between microresonators and dielectric waveguides," Electron. Lett. 36, 321 (2000).

[28] Ghulinyan, M., Guider, R., Pucker, G., and Pavesi, L., "Monolithic whispering-gallery mode resonators with vertically coupled integrated bus waveguides," IEEE Photonics Technol. Lett. 23, 1166-1168 (2011).

[29] Ghulinyan, M., Ramiro-Manzano, F., Prtljaga, N., Guider, R., Carusotto, I., Pitanti, A., Pucker, G., and Pavesi, L., "Oscillatory vertical coupling between a whispering-gallery resonator and a bus waveguide," Phys. Rev. Lett. 110, 163901 (2013).

[30] Ghulinyan, M., Manzano, F. R., Prtljaga, N., Bernard, M., Pavesi, L., Pucker, G., and Carusotto, I., "Intermode reactive coupling induced by waveguide-resonator interaction," Phys. Rev. A 90, 053811 (2014). 